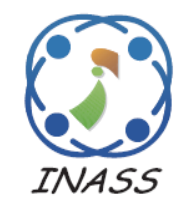

http://www.inass.org/

\title{
Current Research on Glaucoma Detection using Compact Variational Mode Decomposition from Fundus Images
}

\author{
Bhupendra Singh Kirar ${ }^{1 *}$ \\ Dheeraj Kumar Agrawal ${ }^{1}$ \\ ${ }^{I}$ Department of Electronics and Communication Engineering, \\ Maulana Azad National Institute of Technology, Bhopal, India \\ * Corresponding author's Email: bhup17@gmail.com
}

\begin{abstract}
Glaucoma is one of the foremost causes of blindness over the world. It develops slowly and damages optic nerve head. Existing methods of glaucoma detection are expensive and sluggish. Hence quick and low-priced methods are required. In this paper, a novel fully variational and adaptive computer based glaucoma detection using compact variational mode decomposition (CVMD) from fundus images is proposed. Efficient sub band images having narrow fourier bandwidth, clear and sharp boundaries are obtained using CVMD is the key idea of the proposed method. This gives robust estimate of features. Texture feature capture subtle variation and give more information therefore these features are extracted from efficient sub band images and helps to increase the glaucoma detection accuracy. Extracted features are normalized and classified using support vector machine classifier. The obtained classification accuracies are $86.67 \%, 86.67 \%, 85.42 \%$ and $89.18 \%$ for three, five, eight and tenfold cross validation respectively with kernel parameter 2 . The obtained accuracy, sensitivity, specificity, precision and Fmeasure are $89.18 \%, 90 \%, 85 \%, 93.34 \%$ and $89.34 \%$ respectively for tenfold cross validation. Proposed method is found to be better compared to the existing.
\end{abstract}

Keywords: Glaucoma, Pre-processing, Compact variational mode decomposition, Feature extraction, Feature normalization, Support vector machine.

\section{Introduction}

Glaucoma is a critical disorder with in the eye. Intraocular pressure (IOP) increases due to the blockage of drainage structure of the eye. It leads to vision loss and hence blindness [1]. Glaucoma disorder can be classified as primary open angle glaucoma (POAG) and primary angle closure glaucoma (PACG). POAG accounting for at least $90 \%$ of all glaucoma cases [2]. It is estimated that 64.3 million suffered from this disorder in the year 2013. It may reach to 111.8 million by the year 2040 [3]. Glaucoma disorder is $2.5 \%$ for all age's person and $4.8 \%$ for 75 years of ages [1]. About $12.3 \%$ of blindness cases reported over the world are due to glaucoma [4].

Scanning Laser Ophthalmoscope (SLO), Optical Coherence Tomography (OCT), Scanning Laser Polarimeter (SLP), Heidelberg Retina Tomography
(HRT) have been extensively used for glaucoma diagnosis [5]. These are costly and time consuming methods.

Several researchers have done research on glaucoma detection using public database RIM-One [6]. Most of the research is based on the decomposition and extraction of features from fundus images and the use of different types of classifiers.

Raja [7] proposed a glaucoma screening method using trispectrum and complex wavelet transform (CWT) based features. They reported an accuracy of $81 \%$ using artificial neural network (ANN). Raja [8] decomposed images by wavelet packet decomposition (WPD). Entropy and energy features are extracted and ANN yielded an accuracy of $85 \%$. Raja [9] proposed a technique for automated detection of glaucoma using optimal hyper analytic wavelet transform (OHAWT) and support vector 
machine (SVM). They reported accuracy of $85 \%$. Gajbhiye [10] proposed a methodology for glaucoma detection using WPD and moment features. Extracted features were normalized and fed to SVM. They reported an accuracy of $86.57 \%$. Ghosh [11] proposed an automated diagnosis of glaucoma using grid colour moment features and back propagation neural network (BPNN). They reported an accuracy of $87.47 \%$.

Automated diagnosis of glaucoma proposed by Maheshwari [12] used 2dimensional empirical wavelet transform (2DEWT) and correntropy features. Normalized and selected features fed to least squares support vector machine (LS-SVM) classifier. They reported an accuracy of $80.7 \%$ for tenfold cross validation. Further Maheshwari [13] presented a second new method for automated diagnosis of glaucoma using 2dimensional variational mode decomposition (2DVMD). Only green component was used and decomposed to extract entropies \& fractal dimensions (FD) features because it contain more information. Normalized and selected features were classified using LS-SVM. They reported an accuracy of $81.22 \%$ for tenfold cross validation.

Araujo [14] proposed a glaucoma diagnosis using diversity indexes for feature extraction. Features were selected using genetic algorithm and fed to SVM classifier. They reported an accuracy of $86.37 \%$. Kirar [15] proposed a new glaucoma diagnosis approach using third level 2dimensional discrete wavelet transform (2D DWT). Six histogram features namely mean, variance, skewness, kurtosis, energy and entropy were extracted and fed to LS-SVM. They reported an accuracy of $88.3 \%$.

State of the art methods used by Raja, Gajbhiye, Ghosh, Araujo and Kirar are less accurate because these methods are limited to dyadic scale and required predefined basis function. In higher level of decomposition only low frequency sub band is used for next level of decomposition. It creates interference from other nearby frequencies in every sub band. All the above explained methods are not adaptive and can attribute main sub band of decomposed image to dissimilar band and also it contain numerous dissimilar image sub band in the same band. Hence the performance is less accurate. Also method used by Maheshwari [12] is less accurate as it suffered from interference and redundancy due to improper segmentation of image spectrum and design of wavelet filter bank. In this adaptive filter bank sub band images varies. It Fails to separate signal if it is composed of two chirp overlapped in both time and frequency domain.
Further another method used by Maheshwari [13] has a drawback that it suffered from boundary effects and sudden signal onset. Also the limitation of this method is that the performance of the system may deteriorate with a larger and diverse database as well as it required predefined number of mode and does not work out of box.

These methods need to enhance the performance of their system. Therefore enhanced, fast and more accurate methods are needed.

In this paper, a novel fully variational and adaptive computer based glaucoma detection using compact variational mode decomposition (CVMD) from fundus images is proposed. Images are decomposed in to efficient sub band images with narrow fourier bandwidth, clear and sharp boundaries is the key idea of the proposed method. This gives robust estimate of features. Thirteen texture features are extracted from efficient sub band images because texture feature capture subtle variation and give more information. These features are more discriminative thus helps to increase the glaucoma detection accuracy. Extracted features are normalized and classified using LS-SVM. This method achieves an accuracy of $89.18 \%$ in glaucoma detection using fundus images. The obtained accuracy is more than the existing methods. Hence the proposed method is better than the state of art methods. The obtained performances are validated and compared with the existing methods for tenfold cross validation. The comparisons of results are provided in Section five.

The proposed method is better because it decomposes images in to sub band images with narrow fourier bandwidth and smooth boundaries with specific direction and oscillatory characteristic with no interference, no boundary effect and no noise.

This paper has total 6 sections. These next five sections are planned as follow: Input images are given in section two. The proposed methodology is explained in section three. The results are given in section four. Section five discussed the results. The paper is concluded in section and six.

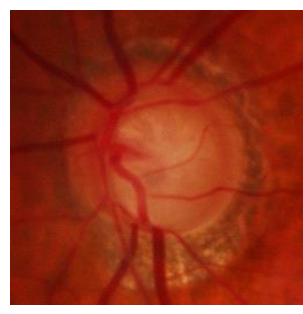

(a)

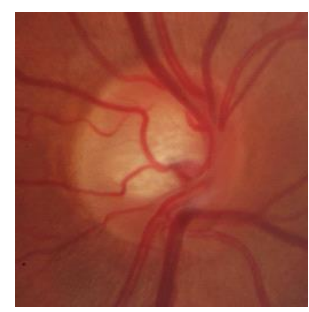

(b)
Figure.1 Input images: (a) glaucoma and (b) healthy 


\section{Input images}

This paper used 30 images (15 glaucoma and 15 normal) of RIM-One Release 1 public database downloaded from Medical Image Analysis Group (MIAG) [6]. Typical glaucoma and healthy images are given in Fig. 1.

\section{Proposed methodology}

The proposed methodology is the third section of this paper. The procedure of proposed current research methodology is given in Fig. 2. It comprise input images, image pre-processing, application of compact variational mode decomposition on preprocessed images, feature extraction from decomposed sub band images, features normalization and classification using support vector machine. The proposed method is validated using three fold, fivefold, eight fold and ten-fold cross validation.

\subsection{Pre-processing}

It is an important step of image processing to remove noise [16]. Input glaucoma and healthy images are pre-processed using resizing ( $256 \times 256)$,

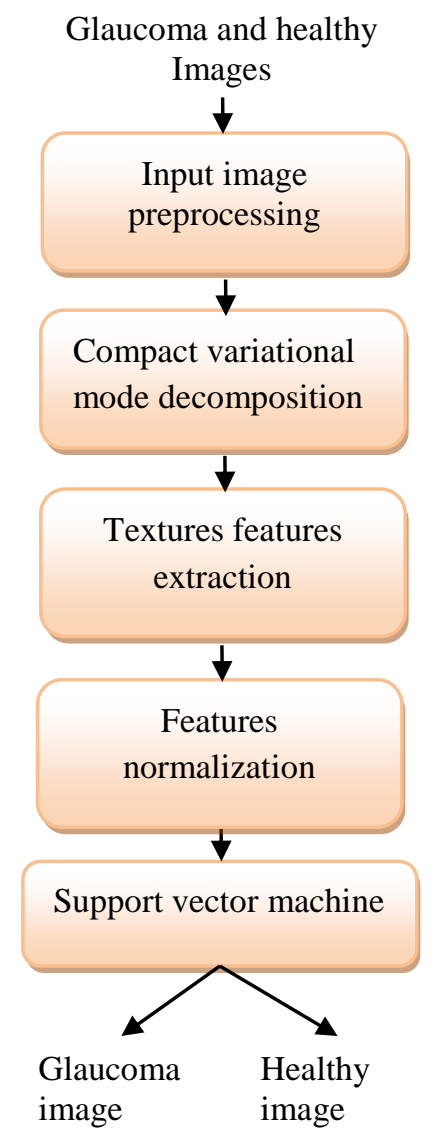

Figure.2 Block diagram of proposed methodology only green channel [1] extraction and applying contrast limited adaptive histogram equalization [17]. Pre-processing made images of same resolution with more information [1] and increased dynamic range.

\subsection{Compact variational mode decomposition}

Compact variational mode decomposition (CVMD) is a spectrally sparse and spatially compact signal (one dimensional or two dimensional) decomposition method [18]. It is a fully adaptive, non-recursive decomposition technique. It is robust to noise and has no boundary distortion and mod mixing problem. The decomposed sub band images are narrow Fourier bandwidth. It allows decomposition of a signal (one dimensional)/image (two dimensional) into modes that may have smooth or sharp boundaries. CVMD is well explained in [18]. The algorithm is explained as follow:

1. Input is defined

2. Initialize for the method and parameters

3. Create 2 dimensional masks for analytic signal Fourier multiplier

4. Retrieve the number of modes

5. Update the number of modes

6. Update the center frequency

7. Defined dual ascent $p-q$ couplong

8. Update binary support function through time split ODE and PDE propagation

9. Rectify binary support function through winner-takes-it-all

10. Define dual ascent data fidelity until convergence.

The complete algorithm is described as [18]:

Input: signal is $f(y), K$ is number of modes, and parameters are: $T, \tau, \tau_{k}, \epsilon, \alpha_{k}, \beta_{k}, \gamma_{k}, \rho, \rho_{k}$

Output: modes $u_{k}(y)$, support functions $B_{k}(y)$ and center frequencies $\omega_{k}$

Initialize $\left\{\omega_{k}\right\},\left\{p_{k}^{0}\right\} \leftarrow 0,\left\{q_{k}^{0}\right\} \leftarrow 0,\left\{B_{k}^{0}\right\} \leftarrow 1$, $\left\{\lambda_{k}\right\}^{0} \leftarrow 0, \lambda^{0} \leftarrow 0, n \leftarrow 0$

repeat the algorithm as given below:

$n \leftarrow n+1$

for $k=1: K$ do

Create 2 dimensional masks for analytic signal Fourier multiplier:

$$
\begin{aligned}
& \mathcal{H}_{k}^{t+1}(\omega) \leftarrow 1+\operatorname{sgn}\left(\left\langle\omega_{k}^{t}, \omega\right\rangle\right) \\
& \text { Update } \hat{p}_{A S, k}: \\
& \hat{p}_{A S, k}^{t+1}(\omega) \leftarrow \mathcal{H}_{k}^{t+1}(\omega)\left[\frac{\rho_{k} \hat{q}_{k}^{t}(\omega)-\widehat{\lambda}_{k}^{t}(\omega)}{\rho_{k}+2 \alpha_{k}\left|\omega-\omega_{k}^{t}\right|^{2}}\right]
\end{aligned}
$$




$$
u_{k}^{t+1}(y) \leftarrow \mathcal{R}\left(\mathcal{F}^{-1}\left\{\hat{p}_{A S, k}^{t+1}(\omega)\right\}\right)
$$

Update $q_{k}$ :

$$
q_{k}^{t+1}(y) \leftarrow \frac{\rho B_{k}^{t}(y)\left(\begin{array}{l}
f(y)-\sum_{i<k} B_{i}^{t}(y) q_{i}^{t+1}(y) \\
-\sum_{i>k} B_{i}^{t}(y) q_{i}^{t}(y)+\frac{\lambda^{t}(y)}{\rho}
\end{array}\right)+}{\rho_{k} p_{k}^{t+1}(y)+\lambda_{k}^{t}(y)}
$$

Update $\omega_{k}$ :

$$
\omega_{k}^{t+1}(y) \leftarrow \frac{\int_{R^{2}} \omega\left|\hat{p}_{A S, p}^{t+1}(\omega)\right|^{2} d \omega}{\int_{R^{2}}\left|\hat{p}_{A S, p}^{t+1}(\omega)\right|^{2} d \omega}
$$

Dual ascent $p-q$ couplong:

$$
\lambda_{k}^{t+1}(y) \leftarrow \lambda_{k}^{t}(y)+\tau_{k}\left(p_{k}^{t+1}(y)-q_{k}^{t+1}(y)\right)
$$

end for

for $k=1: K$ do

Update $B_{k}$ through time split ODE and PDE propagation:

$$
\begin{aligned}
& B_{k}^{t+\frac{1}{3}}(y) \leftarrow \\
& \frac{B_{k}^{t}(y)+T\left(2 \rho q_{k}^{t+1}(y)\left(\begin{array}{c}
-B_{k}^{+} \\
f(y)- \\
\sum_{i<k} B_{i}^{t+\frac{2}{3}}(y) q_{i}^{t+1}(y) \\
-\sum_{i>k} B_{i}^{t}(y) q_{i}^{t+1}(y) \\
+\frac{\lambda^{t}}{\rho}
\end{array}\right)\right)}{1+2 T \rho\left(q_{k}^{t+1}(y)\right)^{2}} \\
& \hat{B}_{k}^{t+\frac{2}{3}}(\omega) \leftarrow \frac{\hat{B}_{k}^{t+\frac{1}{3}}(\omega)}{1+T \gamma_{k}|\omega|^{2}}
\end{aligned}
$$

end for

for $k=1: K$ do

Rectify $B_{k}$ through winner-takes-it-all:

$$
B_{k}^{t+1}(y)= \begin{cases}1 & \text { if } k=\arg \max _{i} B_{i}^{t+\frac{2}{3}}(y) \\ 0 & \text { otherwise }\end{cases}
$$

end for

Dual ascent data fidelity:

$$
\begin{array}{r}
\lambda^{t+1}(y) \leftarrow \lambda^{t}(y)+\tau(f(y)- \\
\left.\sum_{k} B_{k}^{t+1}(y) q_{k}^{t+1}(y)\right)
\end{array}
$$

Until convergence

The meaning of parameters used is given as:
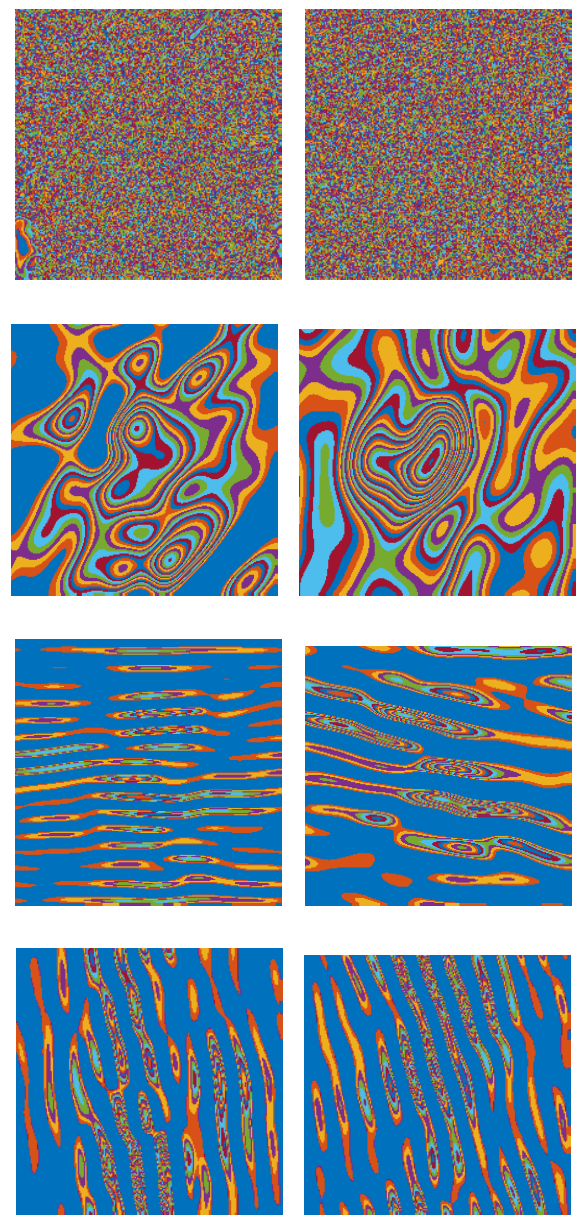

Figure.3 CVMD subs band images: for glaucoma in first column and for healthy in second column

$T$-total time, $\left(\tau, \tau_{k}\right)$-update step size for the Lagrange multipliers, $\epsilon$-typical threshold, $\alpha_{k}$-different weights for $k$ modes, $\beta_{k}$-compact support, $\gamma_{k}$-total variation (TV) weight, $\left(\rho, \rho_{k}\right)$-quadratic penalty coefficients, $f(y)$-input signal, $u_{k}(y)$-output modes, $K$-number of output modes, $\omega_{k}$-center frequencies, $B_{k}(y)$ binary support functions, $\lambda$-Lagrange multipliers,

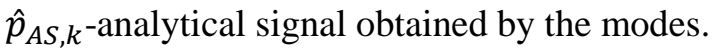

Parameters values used in CVMD are as follow:

Alpha $=1000$, beta $=0.5$, gamma $=500$, rho $=10$, tau $=2.5$, number of mode $=4$, number of iteration $=130$ and first mode at $\mathrm{DC}=1$.

The CVMD sub band images for glaucoma and healthy of Fig. 1 are depicted in Fig. 3. The sub band images for glaucoma are arranged in the first column from top to bottom. Similarly sub band images for healthy image are arranged in the second column from top to bottom. In column top to bottom shows first to fourth CVMD sub band images.

\subsection{Feature extraction and normalization}

Texture features capture subtle variation, give more information and are more discriminative to 
increase the glaucoma detection accuracy therefore total thirteen textures features have been extracted from CVMD sub band images. The extracted features are homogeneity, mean, contrast, correlation, energy, standard deviation, entropy [19], smoothness, root mean square, variance, skewness, kurtosis and inverse difference moment [20].

\subsubsection{Contrast}

It is a measure of intensity within the range 0 and 1 . Any constant image has 0 contrasts.

\subsubsection{Correlation}

It is a measure of joint probability within the range of [-1 1]. It is 1 for positively correlated and 1 for negatively related.

\subsubsection{Energy}

It is defined as the measure of uniformity in the range $[0,1]$. It is 1 for constant image.

\subsubsection{Entropy}

It is the measure of randomness. The larger entropy will have more information and vice versa.

\subsubsection{Homogeneity}

It is a measure of closeness of elements in the GLCM to GLCM diagonal. It's range is [0 1].

\subsubsection{Mean}

It is defined as the measure of average intensity.

\subsubsection{Standard deviation}

It is the square root of the variance or a measure of average contrast.

\subsubsection{Variance}

It is the measure of contrast.

\subsubsection{Kurtosis}

It is the measure of its relative flatness. It is a fourth moment.

\subsubsection{Skewness}

It is third moment and defined as the measure of asymmetry of the data around the mean. It can be positive or negative and zero for the symmetric.

\subsubsection{Smoothness}

It is used to reduce the noise present in an image.

\subsubsection{Inverse different moment}

IDM is also known as homogeneity. It is a measure of closeness as well as texture of image.

\subsubsection{Root mean square}

Root mean square (RMS) is the square root of the mean square. It is also known as the quadratic mean.

The extracted features have different ranges and may affect the performance of the classifiers. Therefore these thirteen features are normalized within the interval $[0,1]$.

\subsection{Support vector machine classifier}

Support vector machine is a supervised learning method $[15,21]$. It is used to classify two or more classes. The proposed method used with least squares (LS) method and radial basis function (RBF) [22]. It is widely used in the field of medical image classification.

Other used classifiers in this paper are: McCallum [23] introduced Naive Bayes multinomial classifier. It is a probabilistic and worked with strong independence assumptions. It is used when the features are dependent on each other. Ibk is an instance-based learning machine which generates predictions for exact instances [24]. Multilayer perceptron is a multiple hidden layer and feed forward neural network. Verma [25] used kNearest Neighbor $(\mathrm{kNN})$ in his work. It is a supervised learning. It classifies data based on nearest neighbors. Random forest is a type of classifier based on ensemble method to constructs forest of random trees [26]. J48 is used to generate unpruned / pruned C4.5 decision tree [27]. Only five performance parameters have been evaluated [28].

\subsubsection{Performance parameters}

The performance parameters; accuracy (ACC), sensitivity (SEN), specificity (SPE), precision (PREC) and F-Measure have been calculated using Eq. (11), Eq. (12), Eq. (13), Eq. (14) and Eq. (15) respectively $[16,29]$.

$$
\begin{aligned}
& A C C=\frac{T P+T N}{T P+T N+F P+F N} \times 100 \\
& S E N=\frac{T P}{T P+F N} \times 100 \\
& S P E=\frac{T N}{T N+F P} \times 100
\end{aligned}
$$




$$
\begin{aligned}
& P R E C=\frac{T P}{T P+F P} \times 100 \\
& \text { FMeasure }=\frac{2|T P|}{2|T P|+|F P|+|F N|} \times 100
\end{aligned}
$$

where, $T P=$ true positive, $T N=$ true negative, $F P=$ false positive and $F N=$ false negative.

\section{Results}

In this paper current research on glaucoma detection using compact variational mode decomposition from fundus images has been proposed. The research method is implemented on 30 images (15glaucoma and 15 normal) of RIM-One Release 1 public image database downloaded from MIAG [6].

Total 13 features have been extracted from 30 images. Thus features dataset of $13 \times 30$ has been obtained. After normalization the obtained dataset is fed to the SVM. We have simulated the proposed method with the following simulation conditions; SVM with least square (LS) method and Radial basis function (RBF). The RBF kernel parameter values from 0.1 to 2.5 (Fig. 4-6), taking features from 1 to 13 (Fig. 7) and cross validations from 2 to 12 (Fig. 8). Best results obtained with kernel parameter $=2$ and taking all 13 features for 10 fold cross validation.

The obtained performance of the proposed method is mention in Table 1 . The obtained accuracy, sensitivity, specificity, precision and Fmeasure are $89.18 \%, 90 \%, 85 \%, 93.34 \%$ and $89.34 \%$ respectively for tenfold cross validation.

Further the kernel parameter is taken from 0.1 to 2.5 with a uniform step size of 0.1. Fig. 4-6 shows the plot of performance versus cross validations.

The obtained accuracy is highest for kernel parameter value $=2$ and tenfold cross validation. Therefore kernel parameter $=2$ and tenfold cross validation has been selected for proposed method.

Table 1. Performance of proposed method (in \%) using

\begin{tabular}{|c|c|c|c|c|}
\hline \multicolumn{1}{c}{ LS-SVM classifier } \\
\hline Parameters & CV $^{* 3}$ & CV5 & CV8 & CV10 \\
\hline Accuracy & 86.67 & 86.67 & 85.4 & 89.18 \\
\hline Sensitivity & 86.67 & 86.67 & 81.25 & 90.00 \\
\hline Specificity & 86.67 & 86.67 & 87.50 & 85.00 \\
\hline Precision & 87.78 & 87.78 & 83.33 & 93.34 \\
\hline F-Measure & 86.59 & 86.59 & 80.83 & 89.34 \\
\hline
\end{tabular}

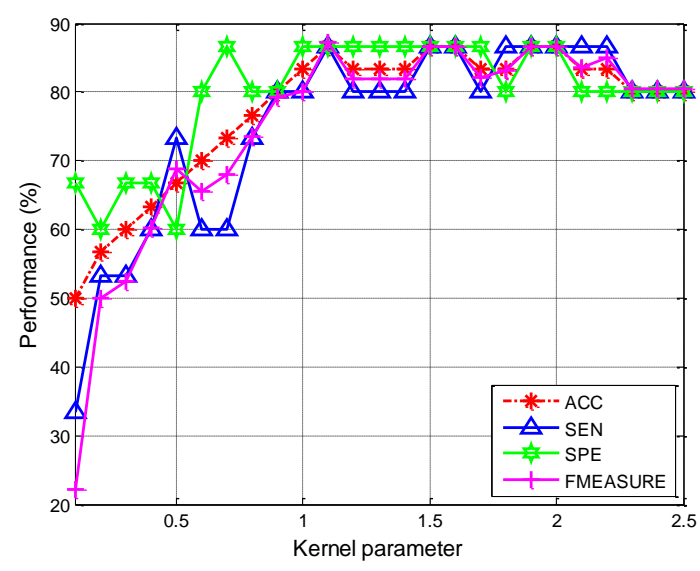

Figure.4 Plot of performance versus kernel parameter for three fold cross validation

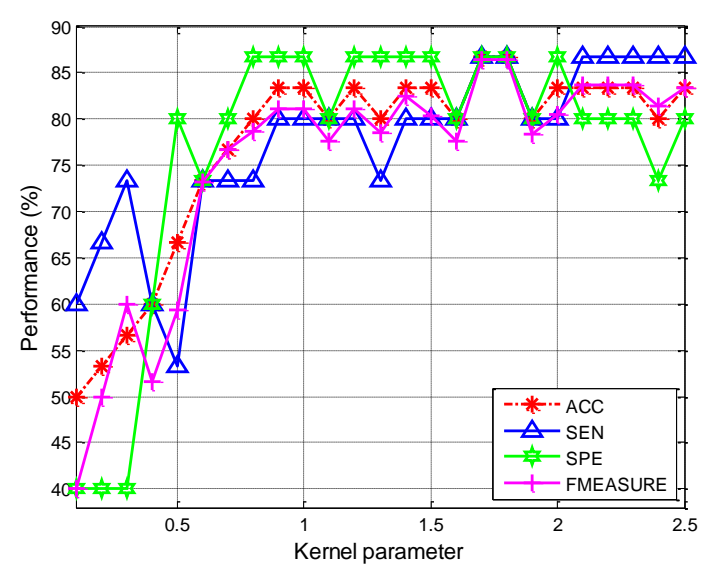

Figure.5 Plot of performance versus kernel parameter for fivefold cross validation

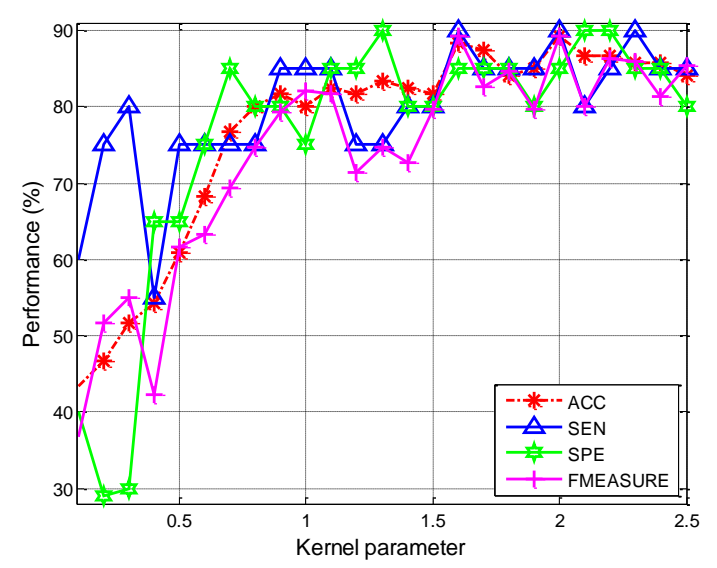

Figure.6 Plot of performance versus kernel parameter for tenfold cross validation

The features are varied from 1 to 13 with kernel parameter $=2$ and tenfold cross validation. Fig. 7 shows the plot of performance versus features. It shows that the obtained accuracy is highest when all 


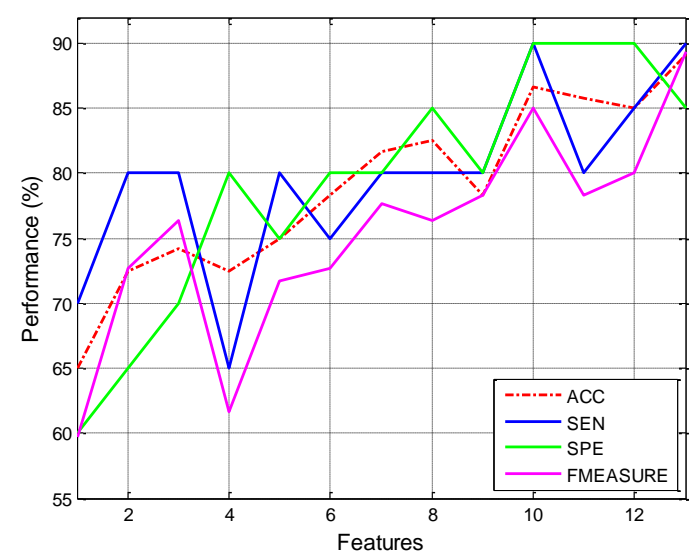

Figure.7 Plot of performance versus features for tenfold cross validation

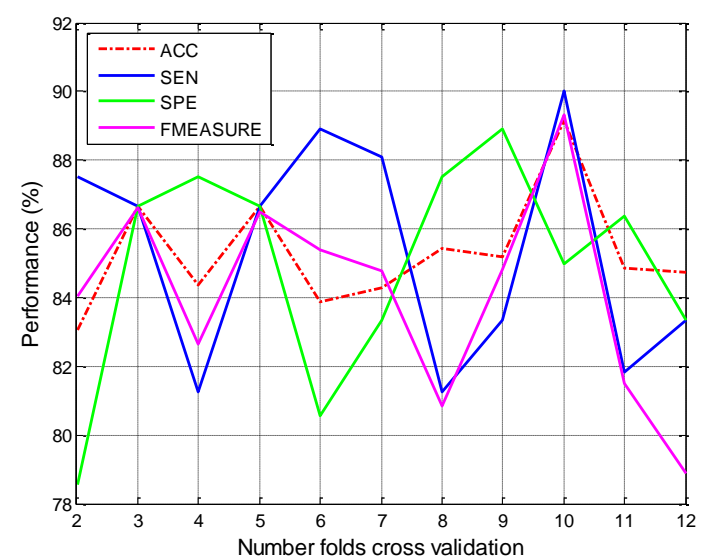

Figure. 8 Plot of performance versus different fold cross validation

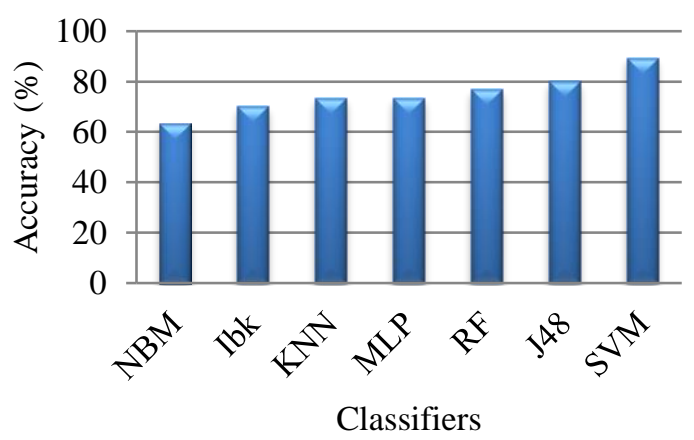

Figure.9 Comparison of accuracy using different classifiers for tenfold cross validation

features are used. Therefore all thirteen features have been selected.

The fold number is varied from 2 to 12 with a step size of 1. Fig. 8 shows the plot of performance versus different fold cross validation for kernel parameter $=2$ and thirteen features. Obtained accuracy is highest for tenfold cross validation. Therefore tenfold cross validation has been selected.

The extracted features also fed to different types of classifiers using weka software to compare the performance of the proposed current research methodology. Fig. 9 confirmed that the selected SVM classifier for the proposed current research methodology yielded highest accuracy.

\section{Discussion}

Current research on glaucoma detection using compact variational mode decomposition from fundus images has been presented. Many researchers have reported on glaucoma detection using decomposition, feature extraction and different types of classifiers from fundus images [6].

Automated screening of glaucoma using trispectrum and CWT based features was proposed by Raja 2013 [7]. They performed the method in 4 steps namely, pre-processing, segmentation, feature extraction and classification. They reported an accuracy, sensitivity and specificity of $81 \%, 87 \%$ and $87 \%$ respectively ANN. Raja [8] in his next work decomposed images using WPD. They extracted entropy and energy features and fed to ANN. The obtained accuracy, specificity and sensitivity are $85 \%, 100 \%$ and $82 \%$ respectively.

Furthermore Raja [9] proposed another technique for automated detection of glaucoma using OHAWT and SVM. The method was performed in 4 steps namely, pre-processing, optimal transformation, feature extraction and classification. They reported an accuracy of $85 \%$.

Gajbhiye [10] proposed a methodology for glaucoma detection using WPD and moment features. They were extracted total 15 features from high-low and high-high decomposed sub band images. These features were normalized using $\mathrm{z}$ score method. SVM, K-Nearest Neighbors (KNN) and Error Back-Propagation Training Algorithm were used for classification. They reported an accuracy of $86.57 \%$ using SVM.

Ghosh [11] proposed an automated diagnosis of glaucoma using grid colour moment features and BPNN. The reported accuracy, sensitivity and specificity are $87.47 \%, 88 \%$ and $87.45 \%$ respectively for tenfold cross validation.

Automated diagnosis of glaucoma proposed by Maheshwari [12] used 2D EWT for image decomposition. Extracted correntropy features are ranked based on $t$ value feature selection method. Then, these selected features are used for the classification of normal and glaucoma images using LS-SVM. The reported accuracy, sensitivity and 
specificity are $80.7 \%, \quad 100 \%$ and $93.33 \%$ respectively for tenfold cross validation.

Maheshwari [13] presented a second new automated glaucoma diagnosis system using 2DVMD. Green component contain more information hence it was decomposed. Images were pre-processed using bicubic interpolation to resize and contrast limited adaptive histogram equalization (CLAHE) to increase dynamic range and contrast of image. Thereafter, 2DVMD applied in iterative manner to decompose the image into sub band images. Entropies \& fractal dimension (FD) features had been extracted. Extracted features were normalized using $\mathrm{z}$-score, selected using reliefF method classified using LS-SVM. They reported accuracy, sensitivity and specificity of $81.22 \%$, $93.62 \%$ and $95.88 \%$ respectively for tenfold cross validation.

Araujo [14] proposed a glaucoma diagnosis using diversity indexes for feature extraction. Features were selected using genetic algorithm and fed to SVM. They have mentions different results but we have taken only analogous to compare our method. The data was prepared using McIntosh index and 31 features and SVM was trained and tested by $60 \%$ and $40 \%$ respectively. They reported an accuracy, sensitivity and specificity of $86.37 \%$, $87.5 \%$ and $85.53 \%$ respectively.

Kirar [15] proposed a glaucoma diagnosis approach in which the pre-processing was applied using images resize to increase the processing speed, only green channel images extraction from resized images as it contains more information, histogram equalization to increase contrast and dynamic range and images are filtered to remove noise. Preprocessed images were decomposed using third level 2D DWT. Six histogram features namely mean, variance, skewness, kurtosis, energy and entropy were extracted and fed to LS-SVM classifier. They reported an accuracy, sensitivity and specificity of $88.3 \%, 90 \%$ and $85 \%$ respectively for tenfold cross validation.

But the state of art methods used by Raja, Gajbhiye, Ghosh, Araujo and Kirar are less accurate because of some limitation due to interference. In higher level of decomposition only low frequency sub band is used for next level of decomposition. It creates interference from other nearby frequencies in every sub band. All the above explained methods are not adaptive and can attribute main sub band of decomposed image to dissimilar band and also it contain numerous dissimilar image sub band in the same band. Further, these methods are limited to dyadic scale and required predefined basis function. Hence the performances are less accurate.

In 2D EWT the performance is less accurate as it suffered from interference and redundancy due to improper segmentation of image spectrum and design of wavelet filter bank. It is adaptive and hence adaptive filter bank leads to vary sub band images. It fails to separate signal if it is composed of two chirp overlapped in both time and frequency domain. 2D VMD, the limitation of this method is that, the performance of the system may deteriorate with a larger and diverse database as well as it required predefined number of mode and does not work out of box.

In this paper, a novel variational and adaptive computer based glaucoma detection using CVMD from fundus images is proposed. Images are decomposed in to efficient sub band images with sharp boundaries. Thirteen texture features have been extracted from efficient sub band images because texture feature capture subtle variation with more information. These features are more discriminative thus helps to increase the glaucoma detection accuracy.

Table 2. Comparison of methods (in \%) for RIM-One MIAG image database

\begin{tabular}{|c|c|c|c|c|c|}
\hline Authors \& ref. & Methods \& features / cross validation & Classifiers & ACC & SEN & SPE \\
\hline Raja [7] & CWT \& HOS / NR & ANN & 81 & 87 & 87 \\
\hline Raja [8] & WPD, entropy \& energy features / NR & ANN & 85 & 82 & 100 \\
\hline Raja [9] & OHAWT / 10 & SVM & 85 & NR & NR \\
\hline Gajbhiye [10] & WPD \& moment Feature / 10 & SVM & 86.57 & NR & NR \\
\hline Gosh [11] & Grid color moment features / 10 & BPNN & 87.47 & 87.5 & 87.45 \\
\hline Maheshwari [12] & 2DEWT and correntropy features / 10 & LS-SVM & 80.66 & 100 & 93.33 \\
\hline Maheshwari [13] & 2DVMD, entropy \& FD feature /10 & LS-SVM & 81.22 & 93.6 & 95.88 \\
\hline Araujo [14] & $\begin{array}{c}\text { McIntosh index using 31 features / training } \\
\text { \& testing (60/40)\% }\end{array}$ & SVM & 86.37 & 87.5 & 85.53 \\
\hline Kirar [15] & DWT and histogram features / 10 & LS-SVM & 88.3 & 90 & 85 \\
\hline Proposed method & CVMD and texture features / 10 & LS-SVM & 89.18 & 90 & 85 \\
\hline
\end{tabular}


Extracted features are normalized and classified using LS-SVM. The obtained results found better than the existing and compared methods as depicted in Table 2. This shows that our method detects glaucoma more accurately and hence it out performed over state of art methods. The obtained performances have been validated and compared with the existing methods for tenfold cross validation using public image database [6]. A detail comparison has been given in Table 2 .

Proposed method found better because the used CVMD method decomposed images in to efficient sub band images with narrow Fourier bandwidth and smooth boundaries no interference, no boundary effect and hence it overcomes the limitations of compared methods.

\section{Conclusion}

This paper presented a current research on glaucoma detection using compact variational mode decomposition from fundus images using LS-SVM classifier. The green channel image is extracted from the digital fundus images and is decomposed using CVMD. The obtained classification accuracy is better for tenfold cross validation with kernel parameter $=2$ and thirteen features. The obtained accuracy, sensitivity, specificity, precision and Fmeasure are $89.18 \%, 90 \%, 85 \%, 93.34 \%$ and $89.34 \%$ respectively for tenfold cross validation. Table 2 and Fig. 9 confirmed that the proposed current research is better than the available methods.

The proposed method was found to be effective in glaucoma detection. It may considerably increase the diagnosis speed of ophthalmologists. The proposed methodology requires testing for huge image database. It can be used for different types of other diseases like diabetes and retinopathy, ovarian cancer, and fatty liver.

\section{Acknowledgments}

Authors want to thank to MIAG [6] for providing fundus images.

\section{References}

[1] R. Bock, J. Meier, L. G. Ny'ul, J. Hornegger, and G. Michelson, "Glaucoma Risk Index: Automated Glaucoma Detection From Color Fundus Images", Medical Image Analysis, Vol.14, No.3, pp.471-481, 2010.

[2] https://www.glaucoma.org/glaucoma/types-ofglaucoma.php (accessed on 2 April 2017).

[3] Y.C. Tham, X.Li, T.Y. Wong, H.A. Quigley, T. Aung, and C.Y. Cheng, "Global Prevalence of
Glaucoma and Projections of Glaucoma Burden Through 2040: A Systematic Review and MetaAnalysis", Ophthalmology, Vol.121, No.11, pp.2081-2090, 2014.

[4] S. Resnikoff, D. Pascolini, D. Etya'ale, I. Kocur, R. Pararajasegaram, G.P. Pokharel, and S.P. Mariotti, "Global Data on Visual Impairment in The Year 2002", Bulletin of the World Health Organization, Vol.82, No.11, 2004.

[5] U.R. Acharya, M.K. Mookiah, J.E.W. Koh, J.H. Tan, S.V. Bhandary, A.K. Rao, H. Fujita, Y. Hagiwara, C.K. Chua, and A. Laude, "Automated Screening System for Retinal Health using Bi-Dimensional Empirical Mode Decomposition and Integrated Index", Computers in Biology and Medicine, Vol.75, pp.54-62, 2016.

[6] http://medimrg.webs.ull.es, (accessed on 25 April 2017).

[7] C. Raja, and N. Gangatharan, "Glaucoma Detection in Fundal Retinal Images using Trispectrum and Complex Wavelet Based Features", European Journal of Scientific Research, Vol.97, No.1, pp. 159-171, 2013.

[8] C. Raja, and N. Gangatharan, "Appropriate Sub-Band Selection in Wavelet Packet Decomposition for Automated Glaucoma Diagnoses", International Journal of Automation and Computing, Vol.12, No.4, pp.393-401, 2015.

[9] C. Raja, and N. Gangatharan, "Optimal Hyper Analytic Wavelet Transform for Glaucoma Detection in Fundal Retinal Images", J Electr Eng Technol., Vol.10, No.4, pp.1899-1909, 2015.

[10] G.O. Gajbhiye, and A.N. Kamthane, "Automatic Classification of Glaucomatous Images Using Wavelet and Moment Feature, In: Proc. of Annual IEEE India Conference (INDICON), New Delhi, pp.1-5, 2015.

[11] A. Ghosh, A. Sarkar, A.S. Ashour, D. BalasTimar, N. Dey, and V.E. Balas, "Grid Color Mo-Ment Features in Glaucoma Classification", International Journal of Advanced Computer Science and Applications (IJACSA), Vol.6, No.9, 2015.

[12] S. Maheshwari, R.B. Pachori, and U.R. Acharya, "Automated Diagnosis of Glaucoma using Empirical Wavelet Transform and Correntropy Features Extracted from Fundus Images", IEEE J. Biomed. Health Inf., Vol.21, No.3, pp.903-813, 2017.

[13] S. Maheshwari, R.B. Pachori, V. Kanhangad, S.V. Bhandary, and U.R. Acharya, "Iterative Variational Mode Decomposition Based 
Automated Detection of Glaucoma using Fundus Images", Comput Biol Med., Vol.88, pp.142-149, 2017.

[14] J.D.L. Araujo, J.C. Souza, O.P.S. Neto J.A.D. Sousa, J.D.S.D. Almeida, A.C.D. Paiva, A.C.E. Silva, G.B. Junior, M. Gattass, "Glaucoma Diagnosis in Fundus Eye Images using Diversity Indexes", Multimed Tools Appl, 2018.

[15] B.S. Kirar, and D.K. Agrawal, "Glaucoma Diagnosis using Discrete Wavelet Transform and Histogram Features from Fundus Image", International Journal of Engineering \& Technology(UAE), Vol.7, No.4, pp.2546-2551, 2018.

[16] A. Manjaramkar, and M. Kokare, "Connected Component Clustering Based Hemorrhage Detection in Color Fundus Images", International Journal of Intelligent Engineering and Systems, Vol.11, No.2, 2018.

[17] K. Zuiderveld, Contrast Limited Adaptive Histograph Equalization, Graphic Gems IV. San Diego, Academic Press Professional, pp.474-485, 1994.

[18] D. Zosso, K. Dragomiretskiy, A.L. Bertozzi, and P.S. Weiss, "Two-Dimensional Compact Variational Mode Decomposition", Journal of Mathematical Imaging and Vision, Vol.58, No.2, pp.294-320, 2017.

[19] B.S. Kirar, and D.K. Agrawal, "Empirical Wavelet Transform Based Preprocessing and Entropy Feature Extraction from Glaucomatous Digital Fundus Images", In: Proc. of International Conference on Recent Innovations in Signal processing and Embedded Systems (RISE), Bhopal, India, pp.315-319, 2017.

[20] R.C. Gonzalez, and R.E. Woods, Digital Image Processing, Third edn., Pearson, UP, India, 2014.

[21] J.A.K. Suykens and J. Vandewalle, "Least Squares Support Vector Machine Classifiers," Neural Processing Letters, Vol.9, No.3, pp.293-300, 1999.

[22] A.H. Khandoker, D.T.H. Lai, R.K. Begg, and M. Palaniswami, "Wavelet Based Feature Extraction for Support Vector Machines for Screening Balance Impairments in The Elderly", IEEE Transactions on Neural Systems and Rehabilitation Engineering, Vol.15, No.4, pp.587-597, 2007.

[23] A. McCallum, and K. Nigam, "A Comparison of Event Models for Naive Bayes Text Classification", In: Proc. of the AAAI-98 workshop on learning for text categorization, pp.41-48, 1998.
[24] D.W. Aha, D. Kibler, and M.K. Albert, "Instance Based Learning Algorithms. Machine Learning, Vol.6, No.1, pp.37-66, 1991.

[25] K.L. Verma, A.K. Jaiswal, M. Mishra, and A.K. Shukla, "EGG Interface Module for Cognitive Assessment Through Neurophysiologic Tests", International Journal of Cognitive Research in Science, Engineering and Education, Vol.2, No.2, 2014.

[26] L. Breiman, "Random forests", Machine Learning, Vol.45, No.1, pp.5-32, 2001.

[27] S.L. Salzberg, C4.5: Programs for Machine Learning by J. Ross Quinlan. Morgan Kaufmann Publishers, Inc., 1993. Machine Learning, Vol.16, No.3, pp.235-240, 1994.

[28] R. Sharma, R.B. Pachori, and U.R. Acharya, "Application of Entropy Measures on Intrinsic Mode Functions for The Automated Identification of Focal Electroencephalogram Signals", Entropy, Vol.17, No.2, pp.669-691, 2015.

[29] H.M. Premalatha, C.V. Srikrishna, "Software Fault Prediction and Classification using Cost based Random Forest in Spiral Life Cycle Model", International Journal of Intelligent Engineering and Systems, Vol.11, No.2, 2018. 